12

\title{
Усовершенствованный цилиндрический зеркальный энергоанализатор
}

\section{() Л.А. Баранова}

Физико-технический институт им. А.Ф. Иофрфе, 194021 Санкт-Петербург, Россия

e-mail: I.baranova@mail.ioffe.ru

(Поступило в Редакцию 29 июня 2016 г.)

\begin{abstract}
Проведено исследование электронно-оптических свойств усовершенствованной конструкции цилиндрического зеркального энергоанализатора. Как внешний, так и внутренний электроды анализатора разделены на три изолированные части каждый, вследствие чего потенциалы на отдельных частях регулируются независимо друг от друга. В симметричном режиме работы при одинаковых потенциалах на боковых частях электродов получено существенное повышение разрешающей способности и светосилы анализатора по сравнению со стандартной конструкцией цилиндрического зеркала. В асимметричном режиме работы, реализуемом при линейном распределении потенциала на внешнем электроде, найдены условия, при которых в несколько раз возрастает линейная дисперсия анализатора.
\end{abstract}

DOI: 10.21883/JTF.2017.03.44255.1962

\section{Введение}

Цилиндрический зеркальный энергоанализатор широко применяется для анализа энергетических спектров заряженных частиц как в научных исследованиях, так и в промышленности. Его первое упоминание в научной литературе относится, по-видимому, к 1957 г. [1]. В работе [2] показано, что в цилиндрическом зеркале может быть осуществлена фокусировка второго порядка по углу влета заряженных частиц. Подробное описание дисперсионных и фокусирующих свойств цилиндрического зеркала приведено в [3]. Показано, что этот прибор обладает высокой светосилой и разрешающей способностью благодаря осевой симметрии и наличию фокусировки второго порядка по углу влета. В 2013 г. опубликована работа [4], в которой предложена простая модификация цилиндрического зеркала, позволяющая существенно улучшить его электронно-оптические характеристики. Внешний электрод модифицированной конструкции разрезан на три электрически изолированных части, потенциалы на которых могут регулироваться независимо. Найдены оптимальные геометрические и электрические параметры модифицированного зеркала, при которых доминирующий коэффициент сферической аберрации уменьшается в несколько раз, что позволяет существенно повысить разрешающую способность анализатора и/или его светосилу. В настоящей работе предложено усовершенствование модифицированной конструкции с целью дальнейшего повышения разрешающей способности и светосилы цилиндрического зеркального энергоанализатора. Кроме того, найдены условия, при которых модифицированная конструкция позволяет существенно увеличить линейную дисперсию анализатора.

\section{Конструкция усовершенствованного цилиндрического зеркала}

Схематическое изображение усовершенствованного цилиндрического зеркала представлено на рис. 1. Каждый из образующих его цилиндрических электродов (как внешний, так и внутренний) разделен на три электрически изолированных части, потенциалы на которых регулируются независимо. В этом отличие от работы [4], где дискретным был только внешний электрод. В [4] была найдена оптимальная конструкция модифицированного зеркала, поэтому здесь мы сохранили те же самые

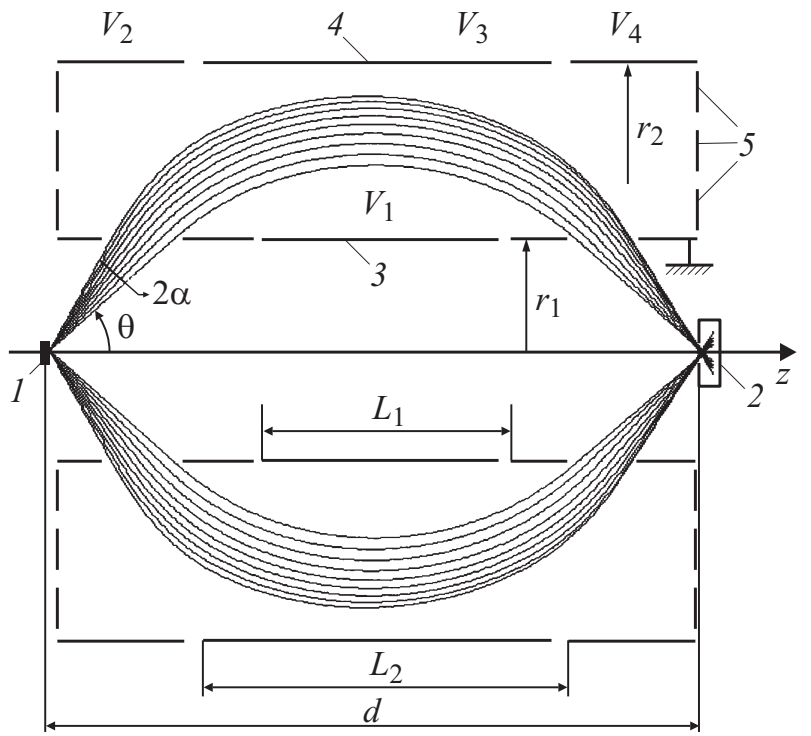

Рис. 1. Схематическое изображение усовершенствованного цилиндрического зеркального анализатора: 1 - исследуемый образец, 2 - детектор, 3 - внутренний цилиндр, 4 внешний цилиндр, 5 - электроды, компенсирующие краевое поле. 
геометрические параметры, за исключением того, что внутренний цилиндр также сделан дискретным. Отношение радиусов внешнего и внутреннего цилиндров составляет $r_{2} / r_{1}=2.6$. Длина центральной части внутреннего цилиндра равна $L_{1} / r_{1}=2.5$, длина центральной части внешнего цилиндра равна $L_{2} / r_{1}=3.5$. Расстояние от источника заряженных частиц до середины каждой из центральных частей, измеренное вдоль оси $z$, составляет $z / r_{1}=3.25$.

\section{Симметричный режим работы усовершенствованного цилиндрического зеркала}

Электронно-оптические свойства цилиндрического зеркального анализатора были исследованы численно с помощью программы CPO3D [5]. Мы ограничились случаем, когда источник заряженных частиц и детектор расположены на оптической оси анализатора и заряженные частицы поступают в поле и выходят из него через окна во внутреннем цилиндре (фокусировка ось-ось). Задача исследования заключалась в том, чтобы найти оптимальные потенциалы на внешнем и внутреннем цилиндре, обеспечивающие наилучшее качество фокусировки. В качестве первого приближения был взят оптимальный режим, найденный в [4] для случая сплошного внутреннего цилиндра, находящегося под нулевым потенциалом. Затем на центральную часть внутреннего цилиндра подавался некоторый потенциал и осуществлялся поиск потенциалов на внешнем цилиндре, обеспечивающих минимальное размытие изображения на оси $z$ - минимальное $\Delta z$. Рассматривались только симметричные режимы, когда потенциалы на боковых частях внешнего цилиндра одинаковы. Потенциалы на боковых частях внутреннего цилиндра всегда оставались равными нулю. Затем процедура повторялась для ряда других значений потенциала на центральной части внутреннего электрода. Из полученных данных был выбран режим, обеспечивающий наилучшую фокусировку в данной конструкции. Оптимальный режим характеризуется следующими значениями потенциалов: потенциал на центральной части внутреннего цилиндра $e V_{1} / E=$ $=-0.098$, потенциал на боковых частях внешнего цилиндра $e V_{2} / E=e V_{4} / E=0.98$, потенциал на центральной части внешнего цилиндра $e V_{3} / E=0.568$, т. е. напряженность поля в центре анализатора меньше напряженности на краях. Потенциал, поданный на центральную часть внутреннего цилиндра, противоположен по знаку потенциалам на внешнем цилиндре.

На рис. 2 показаны траектории заряженных частиц и эквипотенциальные линии в меридиональной плоскости усовершенствованного цилиндрического зеркала. Здесь угол влета заряженных частиц меняется в интервале $31^{\circ}<\theta<61^{\circ}$, т.е. угол захвата составляет $30^{\circ}$ при сохранении острой фокусировки. Такой большой угол захвата является рекордным для анализаторов

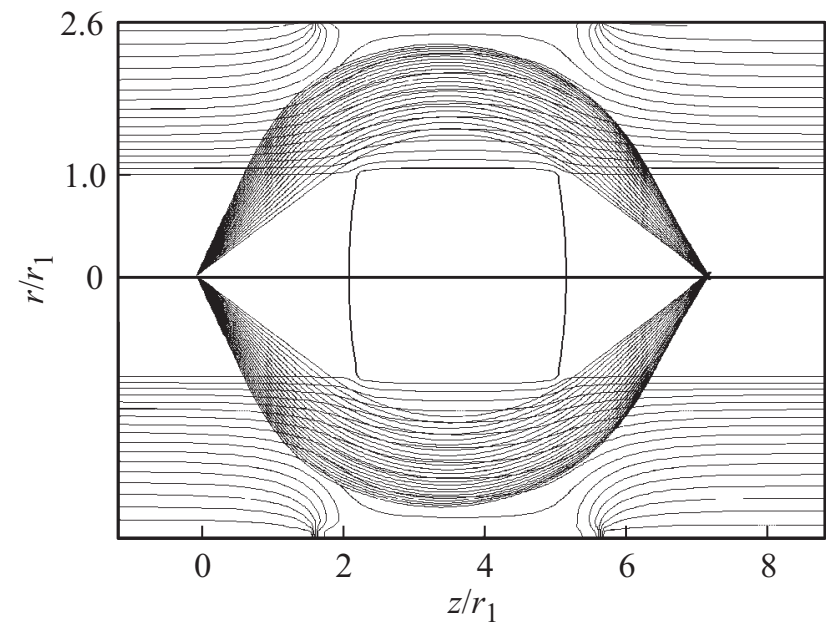

Рис. 2. Эквипотенциальные линии и траектории заряженных частиц в усовершенствованном цилиндрическом зеркальном анализаторе в симметричном режиме работы.

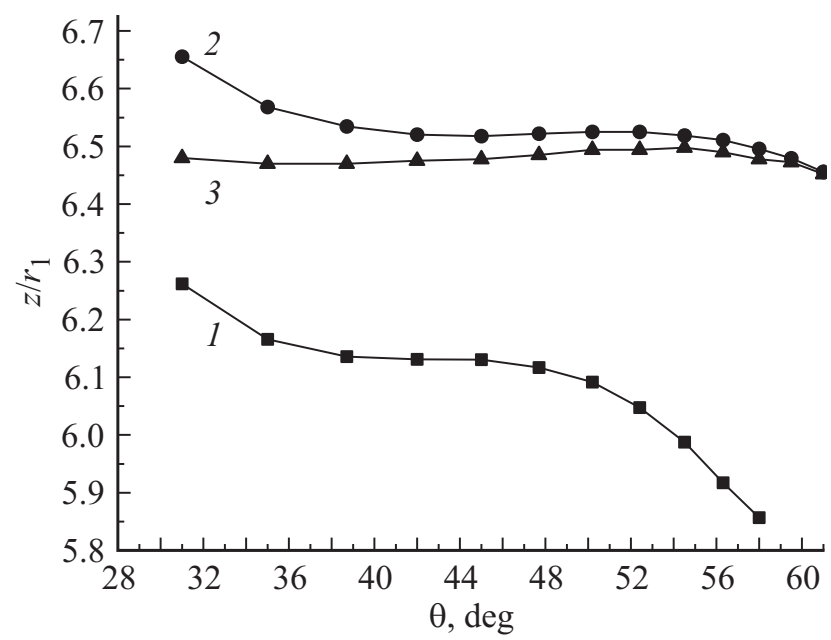

Рис. 3. Координата точки пересечения траектории заряженной частицы с осью $z$ в зависимости от угла входа заряженных частиц $\theta: 1-$ традиционное цилиндрическое зеркало, 2 модифицированное цилиндрическое зеркало, 3 - усовершенствованное цилиндрическое зеркало.

типа цилиндрического зеркала. Следует отметить, что в пространстве, ограниченном внутренним цилиндром, теперь присутствует поле, отличное от нуля, однако оно достаточно мало, чтобы не возмущать траектории вблизи источника и детектора. На практике это поле легко может быть заэкранировано цилиндром меньшего диаметра.

Рис. 3 позволяет сравнить качество фокусировки в традиционном, модифицированном и усовершенствованном цилиндрических анализаторах. На рисунке представлена зависимость координаты пересечения траектории с осью $z$ от угла влета частицы в поле. Усовершенствованное зеркало обеспечивает наибольший интервал углов $\theta$, в котором значение координаты $z$ практически 
Таблица 1. Разрешение $\rho$ трех типов цилиндрического зеркального энергоанализатора в зависимости от угла раствора $2 \alpha$ входящего пучка

\begin{tabular}{c|c|c|c}
\hline $\begin{array}{c}\text { Угол } \\
\text { раствора } \\
\text { пучка } 2 \alpha\end{array}$ & $\begin{array}{c}\text { Стандартное } \\
\text { зеркало, } \\
\text { разрешение } \rho\end{array}$ & $\begin{array}{c}\text { Модифицированное } \\
\text { зеркало, } \\
\text { разрешение } \rho\end{array}$ & $\begin{array}{c}\text { Усовершенствованное } \\
\text { зеркало, } \\
\text { разрешение } \rho\end{array}$ \\
\hline $6^{\circ}$ & 0.001 & 0.0005 & 0.0005 \\
$10^{\circ}$ & 0.003 & 0.0013 & 0.0015 \\
$28^{\circ}$ & 0.07 & 0.03 & 0.0045
\end{tabular}

не зависит от угла влета. Сравнение трех кривых на рис. 3 позволяет сделать вывод, что дискретный внешний цилиндр улучшает фокусировку при больших углах $\theta$, тогда как дискретный внутренний цилиндр корректирует фокусировку при малых углах.

В табл. 1 приведены значения разрешения в зависимости от угла раствора входящего пучка для трех типов цилиндрического зеркала. При углах раствора $2 \alpha=6^{\circ}$ и $2 \alpha=10^{\circ}$ разрешение модифицированного и усовершенствованного анализаторов практически одинаково и вдвое превосходит разрешение стандартного цилиндрического зеркала. При угле раствора $2 \alpha=28^{\circ}$ разрешение усовершенствованного зеркала более, чем на порядок лучше разрешения стандартного и примерно в 7 раз превосходит разрешение модифицированного зеркал. Следует отметить, что подбор потенциалов при настройке наилучшего качества изображения производился вручную, что не гарантирует того, что найден абсолютный минимум размытия изображения. Использование компьютера для автоматизации этого процесса возможно позволит найти режимы с лучшими параметрами.

\section{Асимметричный режим работы модифицированного цилиндрического зеркала}

В работе [6], где исследовались свойства цилиндрического зеркала при запуске заряженных частиц через торцевую диафрагму, было показано, что с помощью независимой регулировки потенциалов на отдельных частях внешнего цилиндра можно добиться существенного увеличения линейной дисперсии анализатора. В настоящей работе исследована возможность увеличения линейной дисперсии при запуске заряженных частиц через окно во внутреннем цилиндре в режиме фокусировки „ось-ось“( (стандартный режим работы цилиндрического зеркала). Конструкция анализатора оставалась такой же, как представленная на рис. 1. Расчеты показали, что увеличение дисперсии происходит в асимметричном режиме работы, когда напряженность поля анализатора убывает в направлении от источника заряженных частиц к детектору. При этом варьирование потенциала на центральной части внутреннего цилиндра не приводит к какому-либо положительному результату, поэтому в дальнейшем рассматривался сплошной внутренний цилиндр под нулевым потенциалом. На левую боковую часть внешнего цилиндра (ближайшую к источнику) подавался постоянный потенциал $V_{2}$. От левого края центральной части внешнего цилиндра до правого края его боковой части (ближайшей к детектору) подавался потенциал, линейно меняющийся с координатой $z$ от значения $V_{3}$ до значения $V_{4}$ (программа CPO3D позволяет задавать такие граничные условия). Исследование осуществлялось следующим образом. Задавалось некоторое значение потенциала $V_{4}$ и проводился поиск потенциалов $V_{2}$ и $V_{3}$, обеспечивающих фокусировку второго порядка на оси анализатора. Затем процедура повторялась для других значений потенциала $V_{4}$. Как оказалось, фокусировки второго порядка можно достичь при различных соотношениях потенциалов $V_{2}$ и $V_{3}$, в дальнейшем для простоты мы ограничились случаем $V_{2}=V_{3}$.

Табл. 2 иллюстрирует зависимость приведенной линейной дисперсии $D / r_{1}$ цилиндрического анализатора от потенциалов на внешнем цилиндре. Первая строка табл. 2 относится к традиционному режиму работы цилиндрического зеркала, когда внешний электрод находится под постоянным потенциалом. Последующие строки расположены в порядке возрастания абсолютной величины потенциала $V_{4}$. Как видно из таблицы, линейная дисперсия возрастает с увеличением градиента электрического поля в направлении оси $z$. Существенное возрастание дисперсии происходит только в том случае, когда потенциал $V_{4}$ противоположен по

Таблица 2. Линейная дисперсия модифицированного цилиндрического зеркала в асимметричном режиме работы в зависимости от потенциалов на отдельных частях внешнего цилиндра

\begin{tabular}{c|c|c|c}
\hline$e V_{2} / E$ & $e V_{3} / E$ & $e V_{4} / E$ & $D / r_{1}$ \\
\hline 0.73 & 0.73 & 0.73 & 5.6 \\
\hline \multicolumn{3}{c}{ Линейное распределение } \\
\hline 0.83 & 0.83 & 0 & 9.2 \\
0.88 & 0.88 & -0.4 & 12.1 \\
0.90 & 0.90 & -0.75 & 20.0 \\
0.903 & 0.903 & -0.77 & 21.1
\end{tabular}




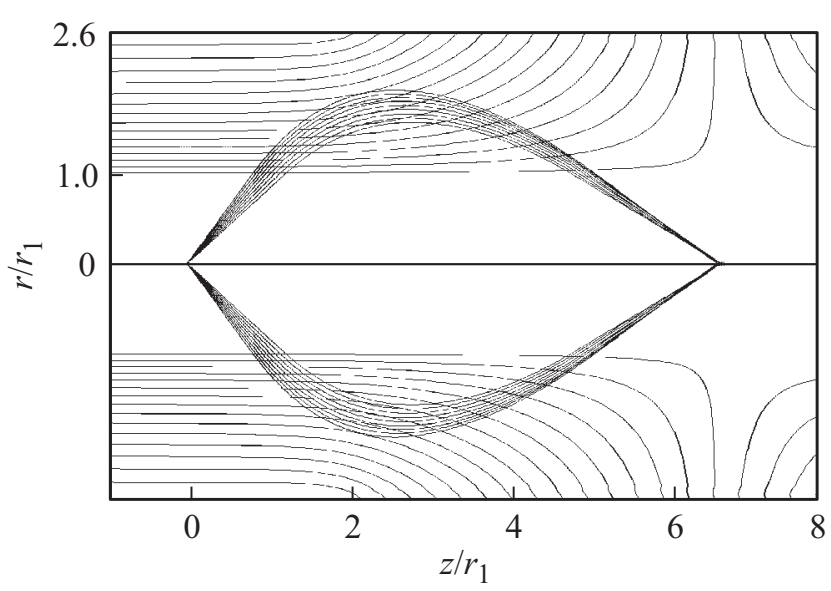

Pис. 4. Эквипотенциальные линии и траектории заряженных частиц в модифицированном цилиндрическом зеркальном анализаторе в асимметричном режиме работы.

знаку потенциалу $V_{3}$. Существует предельное значение абсолютной величины $V_{4}$, превышение которой не позволяет добиться фокусировки второго порядка на оси. В рассматриваемой конструкции предельное значение составляет $\left|e V_{4} / E\right|=0.77$. При этом значении потенциала $V_{4}$ достигается максимальное значение линейной дисперсии, которое почти в 4 раза превосходит дисперсию стандартного цилиндрического зеркала.

Рис. 4 иллюстрирует траектории заряженных частиц и эквипотенциальные линии в меридиональной плоскости модифицированного зеркального энергоанализатора в асимметричном режиме работы. Здесь угол раствора пучка заряженных частиц составляет $2 \alpha=10^{\circ}$. Качество фокусировки в асимметричном режиме работы снижается по сравнению с симметричным режимом. При угле раствора пучка $2 \alpha=10^{\circ}$ разрешение составляет $\rho=0.0018$, т. е. сравнимо с разрешением усовершенствованного зеркала в симметричном режиме, при больших углах раствора разрешение цилиндрического зеркала в асимметричном режиме резко падает.

\section{Заключение}

В работе проведено компьютерное моделирование усовершенствованной конструкции цилиндрического зеркального энергоанализатора. Как внешний, так и внутренний цилиндры усовершенствованной конструкции разделены на три электрически изолированные части каждый, так что потенциалы на отдельных частях могут регулироваться независимо. Варьирование потенциалов на отдельных частях электродов анализатора позволяет в широких пределах изменять его электроннооптические свойства. Показано, что в симметричном режиме работы, когда потенциалы на боковых частях электродов равны между собой, уменьшение напряженности электрического поля в центральной части анализа- тора приводит к существенному росту его разрешающей способности и светосилы.

Найдены потенциалы на электродах анализатора, обеспечивающие оптимальные значения его электроннооптических характеристик. При угле раствора входящего пучка $2 \alpha=6^{\circ}$ разрешение усовершенствованного анализатора вдвое превосходит разрешение стандартного цилиндрического зеркала. При угле раствора $2 \alpha=28^{\circ}$ разрешение усовершенствованного зеркала в 15 раз лучше разрешения стандартного.

Показано, что в асимметричном режиме работы, когда напряженность электрического поля падает в направлении от источника заряженных частиц к детектору, возрастает линейная дисперсия анализатора. В оптимальном режиме работы линейная дисперсия усовершенствованного цилиндрического зеркала в 4 раза превосходит дисперсию стандартного зеркала.

\section{Список литературы}

[1] Blauth E. // Zs. Phys. 1957. Vol. 147. P. 228-231.

[2] Зашквара В.В., Корсунский М.И., Космачев О.С. // ЖТФ. 1966. T. 36. C. $132-138$.

[3] АЯанасьев В.П., Явор С.Я. Электростатические энергоанализаторы для пучков заряженных частиц. М.: Наука, 1978. $224 \mathrm{c}$.

[4] Баранова Л.А. // ЖТФ. 2013. Т. 83. Вып. 4. С. 149-151.

[5] Электронный ресурс. Режим доступа: CРО programs, available from www.electronoptic.com

[6] Баранова Л.А. // ЖТФ. 2016. Т. 86. Вып. 8. С. 153-155. 